\title{
Preoperative Advance Care Planning for Older Adults Undergoing High-Risk Surgery: An Essential but Underutilized Aspect of Clinical Care
}

Kalbfell E, Kata A, Buffington AS, et al. Frequency of Preoperative Advance Care Planning for Older Adults Undergoing High-risk Surgery: A Secondary Analysis of a Randomized Clinical Trial. JAMA Surg. 2021;156(7):e211521. doi:10.1001/jamasurg.2021.1521

\section{Study Overview}

Objective. The objectives of this study were to (1) quantify the frequency of preoperative advance care planning (ACP) discussion and documentation for older adults undergoing major surgery in a national sample, and (2) characterize how surgical patients and their family members considered ACP after postoperative complications.

Design. A secondary analysis of data from a multisite randomized clinical trial testing the effects of a question prompt list intervention (a Question Problem List [QPL] brochure with 11 questions) given to patients aged 60 years or older undergoing high-risk surgery on preoperative communication with their surgeons.

Setting and participants. This multisite randomized controlled trial involved 5 study sites that encompassed distinct US geographic areas, including University of Wisconsin Hospital and Clinics (UWHC), Madison; the University of California, San Francisco, Medical Center (UCSF); Oregon Health \& Science University (OHSU), Portland; the University Hospital of Rutgers New Jersey Medical School (Rutgers), Newark; and the Brigham and
Women's Hospital (BWH), Boston, Massachusetts. The study enrolled 40 surgeons who routinely performed high-risk oncological or vascular surgery via purposeful sampling; patients aged 60 years or older with at least 1 comorbidity and an oncological or vascular problem that were treatable with high-risk surgery; and 1 invited family member per enrolled patient to participate in open-ended interviews postsurgery. High-risk surgery was defined as an operation that has a 30-day in-hospital mortality rate greater than or equal to $1 \%$. Data were collected from June 1, 2016, to November 30, 2018.

Main outcome measures. The frequency of preoperative discussions and documentation of ACP was determined. For patients who had major surgery, any mention of ACP (ie, mention of advance directive [AD], health care power of attorney, or preference for limitations of life-sustaining treatments) by the surgeon, patient or family member during the audio recorded, transcribed, and coded preoperative consultation was counted. The presence of a written $A D$ in the medical record at the time of the initial consultation, filed between the consultation and the date of surgery, or added postoperatively, was recorded

\section{Outcomes Research in Review SECTION EDITORS}

KatRina F. MATEo, PhD, MPH

CUNY School of Public Health New York, NY
DANIEL ISAAC, DO, MS

Michigan State University

East Lansing, Ml
FRED Ko, MD, MS

Icahn School of Medicine at Mount Sinai New York, NY
TAISHI HIRAI, MD

University of Missouri

Columbia, $\mathrm{MO}$
WiLliam W. Hung, MD, MPH

Icahn School of Medicine at Mount Sinai New York, NY 
using a standardized abstraction form. Postoperative treatments administered and complications experienced within 6 weeks after surgery were recorded. Open-ended interviews with patients who experienced significant postoperative complications (eg, prolonged hospitalization $>8$ days, intensive care unit stay $>3$ days) and their family members were conducted 6 weeks after surgery. Information ascertained during interviews focused on treatment decisions, postoperative experiences, and interpersonal relationships among patients, families, and clinicians. Transcripts of these interviews were then subjected to qualitative content analysis.

Main results. A total of 446 patients were enrolled in the primary study. Of these patients, 213 (122 men [57\%]; 91 women [43\%]; mean [SD] age, 72 [7] years) underwent major surgery. Only $13(6.1 \%)$ of those who had major surgery had any discussion related to ACP in the preoperative consultation. In this cohort, 141 (66\%) patients did not have an $A D$ on file before undergoing major surgery. The presence of $A D$ was not associated with age (60-69 years, 26 [31\%]; 70-79 years, 31 [33\%]; $\geq 80$ years, 15 [42\%]; $P=.55$ ), number of comorbidities (1, 35 [32\%]; 2, 18 [33\%]; $\geq 3,19$ [40\%]; $P=.62$ ), or type of procedure (oncological, 53 [32\%]; vascular, 19 [42\%]; $P=$.22). Moreover, there was no difference in preoperative communication about ACP or documentation of an $A D$ for patients who were mailed a QPL brochure compared to those who received usual care (intervention, 38 [35\%]; usual care, 34 [33\%]; $P=.77$ ). Rates of AD documentation were associated with individual study sites with $\mathrm{BWH}$ and UWHC having higher rates of documentation (20 [50\%] and 27 [44\%], respectively) compared to OHSU, UCSF, or Rutgers (7 [17\%], 17 [35\%], and 1 [5\%], respectively). Analysis from the interviews indicated that patients and families felt unprepared for serious surgical complications and had varied interpretations of ACP. Patients with complications were enthusiastic about ACP but did not think it was important to discuss their preferences for life-sustaining treatments with their surgeon preoperatively.

Conclusion. Although surgeons and patients report that they believe ACP is important, preoperative discussion of patient preferences rarely occurs. This study found that the frequency of ACP discussions or AD documentations among older patients undergoing high-risk oncologic or vascular surgery was low. Interventions that are aimed to increase rates of preoperative ACP discussions should be implemented to help prepare patients and their families for difficult decisions in the setting of serious surgical complications and could help decrease postoperative conflicts that result from unclear patient care goals.

\section{Commentary}

Surgeons and patients approach surgical interventions with optimistic outlooks while simultaneously preparing for unintended adverse outcomes. For patients, preoperative ACP discussions ease the burden on their families and ensure their wishes and care goals are communicated. For surgeons, these discussions inform them how best to support the values of the patient. Therefore, it is unsurprising that preoperative ACP is viewed favorably by both groups. Given the consensus that ACP is important in the care of older adults undergoing high-risk surgery, one would assume that preoperative ACP discussion is a standard of practice among surgeons and their aging patients. However, in a secondary analysis of a randomized control trial testing a patient-mediated intervention to improve preoperative communication, Kalbfell et al ${ }^{1}$ showed that ACP discussions rarely take place prior to major surgery in older adults. This finding highlights the significant discrepancy between the belief that ACP is important, and the actual rate that it is practiced, in older patients undergoing high-risk surgery. This discordance is highly concerning because it suggests that surgeons who provide care to a very vulnerable subset of older patients may overlook an essential aspect of preoperative care and therefore lack a thorough and thoughtful understanding of the patient's care goals. In practice, this omission can pose significant challenges associated with the surgeon and family's decisions to use postoperative life-sustaining interventions or to manage unforeseen complications should a patient become unable to make medical decisions.

The barriers to conducting successful ACP discussions between surgeons and patients are multifactorial. Kalbfell et $\mathrm{al}^{1}$ highlighted several of these barriers, 


\section{Outcomes Research in Review}

including lack of patient efficacy, physician attitudes, and institutional values in older adults who require major surgeries. The inadequacy of patient efficacy in preoperative ACP is illustrated by findings from the primary, multisite trial of QPL intervention conducted by Schwarze et al. Interestingly, the authors found that patients who did not receive QPL brochure had no ACP discussions, and that QPL implementation did not significantly improve discussion rates despite its intent to encourage these discussions. ${ }^{2}$ Possible explanations for this lack of engagement might be a lack of health literacy or patient efficacy in the study population. Qualitative data from the current study provided further evidence to support these explanations. For instance, some patients provided limited or incomplete information about their wishes for health care management while others felt it was unnecessary to have ACP discussions unless complications arose. ${ }^{1}$ However, the latter example counters the purpose of ACP which is to enable patients to make plans about future health care and not reactive to a medical complication or emergency.

Surgeons bear a large responsibility in providing treatments that are consistent with the care goals of the patient. Thus, surgeons play a crucial role in engaging, guiding, and facilitating ACP discussions with patients. This role is even more critical when patients are unable or unwilling to initiate care goal discussions. Physician attitudes towards ACP, therefore, greatly influence the effectiveness of these discussions. In a study of selfadministered surveys by vascular, neurologic, and cardiothoracic surgeons, greater than $90 \%$ of respondents viewed postoperative life-supporting therapy as necessary, and $54 \%$ would decline to operate on patients with an $A D$ limiting life-supporting therapy. ${ }^{3}$ Moreover, the same study showed that $52 \%$ of respondents reported discussing $A D$ before surgery, a figure that exceeded the actual rates at which ACP discussions occur in many other studies. In the current study, Kalbfell et $\mathrm{al}^{1}$ also found that surgeons viewed ACP discussions largely in the context of $A D$ creation and declined to investigate the full scope of patient preferences. These findings, when combined with other studies that indicate an incomplete understanding of ACP in some surgeons, suggest that not all physicians are able or willing to navigate these sometimes lengthy and difficult conversations with patients. This gap in practice provides opportunities for training in surgical specialties that center on optimizing preoperative ACP discussions to meet the care needs of older patients.

Institutional value and culture are important factors that impact physician behavior and the practice of ACP discussion. In the current study, the authors reported that the majority of ACP discussions were held by a minority of surgeons and that different institutions and study sites had vastly different rates of ACP documentation. ${ }^{1}$ These results are further supported by findings of large variations between physicians and hospitals in ACP reporting in hospitalized frail older adults. ${ }^{4}$ These variations in practices at different institutions suggest that it is possible to improve rates of preoperative ACP discussion. Reasons for these differences need to be further investigated in order to identify strategies, resources, or trainings required by medical institutions to support surgeons to carry out ACP discussions with patients undergoing high-risk surgeries.

The study conducted by Kalbfell et al ${ }^{1}$ has several strengths. For example, it included Spanish-speaking patients and the use of a Spanish version of the QPL intervention to account for cultural differences. The study also included multiple surgical specialties and institutions and captured a large and national sample, thus making its findings more generalizable. However, the lack of data on the duration of preoperative consultation visits in patients who completed ACP discussions poses a limitation to this study. This is relevant because surgeon availability to engage in lengthy ACP discussions may be limited due to busy clinical schedules. Additional data on the duration of preoperative visits inclusive of a thoughtfully conducted ACP discussion could help to modify clinical workflow to facilitate its uptake in surgical practices.

\section{Applications for Clinical Practice}

The findings from the current study indicate that patients and surgeons agree that preoperative ACP discussions are beneficial to the clinical care of older adults before high-risk surgeries. However, these important conversations do not occur frequently. Surgeons and health care institutions need to identify strategies to initiate, facilitate, and optimize productive preoperative ACP discussions 
to provide patient-centered care in vulnerable older surgical patients.

-lan Chun, BS, John A. Burns School of Medicine, University of Hawaii, Honolulu, HI, and Fred Ko, MD

Financial disclosures: None. doi:10.12788/jcom.0066

\section{References}

1. Kalbfell E, Kata A, Buffington AS, et al. Frequency of Preoperative Advance Care Planning for Older Adults Undergoing High-risk
Surgery: A Secondary Analysis of a Randomized Clinical Trial. JAMA Surg. 2021;156(7):e211521. doi:10.1001/jamasurg.2021.1521

2. Schwarze ML, Buffington A, Tucholka JL, et al. Effectiveness of a Question Prompt List Intervention for Older Patients Considering Major Surgery: A Multisite Randomized Clinical Trial. JAMA Surg. 2020;155(1):6-13. doi:10.1001/jamasurg.2019.3778

3. Redmann AJ, Brasel KJ, Alexander CG, Schwarze ML. Use of advance directives for high-risk operations: a national survey of surgeons. Ann Surgery. 2012;255(3):418-423. doi:10.1097/ SLA.0b013e31823b6782

4. Hopkins SA, Bentley A, Phillips V, Barclay S. Advance care plans and hospitalized frail older adults: a systematic review. BMJ Support Palliat Care. 2020;10:164-174. doi:10.1136/ bmjspcare-2019-002093

\section{Differences in Care by Race in Older Nursing Home Residents With Dementia}

Temkin-Greener H, Yan D, Wang S, Cai S. Racial disparity in end-of-life hospitalizations among nursing home residents with dementia. J Am Geriatr Soc. 2021;69(7):1877-1886. doi:10.1111/jgs. 17117

\section{Study Overview}

Objective. To examine differences in care, specifically hospitalization towards the end of life, among nursing home residents with dementia who were Black compared with those who were White.

Design. Population based cohort study in the US. The study included all decedents with Alzheimer's disease or related dementia (ADRD) who resided in a nursing home from 2014 to 2017. Decedents from nursing homes were identified by death within 1 day of an identified nursing home stay or within 8 days of a hospital transfer from nursing home. Data were obtained from Minimum Data Set 3.0 (MDS) which contains clinical data from all Medicaid or Medicare certified nursing homes, and from the Medicare Beneficiary Summary File (MBSF) and Medicare Provider and Analysis and Review (MedPAR) which contains hospitalization events for all Medicare Beneficiaries. These files were linked to identify nursing home residents with ADRD who were hospitalized at the end of life. ADRD diagnosis was identified from the chronic condition list from the MBSF and from MDS diagnosis list.
Setting and participants. The study included 665033 residents from 14595 nursing homes who died during the study period. Resident race was categorized as White or Black based on the MBSF. Severe cognitive impairment was identified using the MDS that categorized residents as severe or not using the Brief Interview for Mental Status and the Cognitive Performance Scale. The mean (SD) age of the study population was 86.7 (9.2) years for White residents and 82.6 (11.1) years for Black residents. Of the participants, $68.8 \%$ and $61.2 \%$ were female for Black and White residents, respectively. Approximately $23.4 \%$ of White and $32.5 \%$ of Black residents had severe cognitive impairment. For nursing home characteristics, $71.5 \%$ of the 14595 nursing homes represented were for profit; average bedside was 109.5 (57.0) and occupancy rate was on average $81.2 \%$ (14.3\%).

Main outcome measures. The study outcome measure was any hospitalization within 30 days prior to death. The outcome was selected as an indicator of quality of care because as older adults living with ADRD experience progressive worsening of cognitive symptoms, at the end of life when dementia is severe, advance care planning and communication with health care proxies 


\section{Outcomes Research in Review}

and surrogates often result in coordinated care that avoids acute hospitalizations, which are often burdensome to both patient and family and may yield poorer quality of life.

Main results. The study found that approximately 29.5\% of White decedents and $40.7 \%$ of Black decedents were hospitalized towards the end of life. Nursing homes with a higher proportion of Black residents were more likely to have residents hospitalized towards the end of life with $35 \%$ of residents hospitalized in the highest quartile (27\% Black) compared with 17\% hospitalized for nursing homes in the lowest quartile (0\% Black). After adjusting for covariates, Black residents were 7.9\% more likely to be hospitalized in the last 30 days of life compared with White residents. Blacks with severe cognitive impairment has elevated risk of hospitalization by $4.9 \%$ when compared with White residents. After accounting for nursing home facility-level characteristics, nursing homes with a low proportion of Black residents had a 5.2\% higher risk of hospitalizations compared with nursing homes with no Black residents, and nursing homes with a higher percentage of Black residents had a $13.3 \%$ higher risk of hospitalization compared with nursing homes with no Black residents.

Conclusion. Race is associated with care disparities in older nursing home residents with dementia. This study suggests that hospitalization towards the end of life as a quality of care marker differs across nursing homes, and nursing homes with a higher proportion of Black residents were more likely to be hospitalized. This suggests that these nursing homes may have fewer resources and delivered poorer quality of care, and that disparities in health systems or institutions contribute to differences in quality of care for this vulnerable group.

\section{Commentary}

Disparities of health status, health care, and affordability across race and ethnicity have persisted throughout the past 20 years. ${ }^{1}$ There is further evidence to support systemic differences that can contribute to differences in health outcomes. ${ }^{2}$ Although changes in health care policy such as the Affordable Care Act have expanded health care coverage, and instituted changes that aims to improve health care quality and reduce disparities, it is clear that factors contributing to disparities in care are structural and perhaps systemic. The latest evidence comes in this study that examines racial disparities in health care quality in one of the most vulnerable populations-older adults with Alzheimer's disease and dementia. The finding that Black nursing home residents, when compared with White residents, often has higher risk of hospitalization at the end of life, even among those with severe dementia where better coordinated care, clear goals of care and perhaps instituting palliative care would result in lower rate of hospitalization. The disparities were observed across nursing homes as well, where nursing homes with higher proportion of Black residents appear to have lower quality of care.

These findings are consistent with prior work that has examined differences in Black and White population on uptake of palliative care, discussion, and the documentation of advance care planning. ${ }^{3}$ Factors that may contribute to these differences include mistrust of the health care system among minorities, and not being connected to adequate health care resources. Family members and surrogate health care decision makers may consider receiving more aggressive care as advocating for better health care for their family members. ${ }^{4}$ These differences may contribute to the differences in hospitalization rates among residents within the same nursing home; however, the differences between nursing homes even after accounting for individual differences may indicate more widespread systemic differences that is associated with race. Policy changes that will address these differences are needed to level these differences so that quality care can be delivered regardless of race. ${ }^{5}$ For this vulnerable population with a terminal illness, approaches to enhance uptake of palliative approaches and care delivery for dementia patients at terminal stage are needed and understanding and targeting factors that contribute to low uptake of these approaches will enhance end of life care. Understanding the differences in resources and systems of care in nursing homes and perhaps how palliative care is integrated in these settings will be important to address care disparities that occurs across nursing homes. 


\section{Applications for Clinical Practice}

Clinicians who take care of this population of older adults with advanced dementia should be aware of the potential for racial disparities that may lead to differences in the quality of care. The underlying reasons for these differences could be targeted so that older adults in all racial groups may have equal access to quality care including palliative approaches that avoid aggressive care for terminal illnesses across settings that may yield better care and quality of life. Policy makers and health systems leaders need to consider the current realities with racial disparities that policies need to address these differences so that they may not continue to persist in our systems of care.

\section{-William W. Hung, MD, MPH \\ Financial disclosures: None.}

doi:10.12788/jcom.0067

\section{References}

1. Mahajan S, Caraballo C, Lu Y, et al. Trends in Differences in Health Status and Health Care Access and Affordability by Race and Ethnicity in the United States, 1999-2018. JAMA. 2021;326(7):637648. doi:10.1001/jama.2021.9907

2. GillTM,Zang EX,MurphyTE, etal.AssociationBetweenNeighborhood Disadvantage and Functional Well-being in Community-Living Older Persons. [published online ahead of print, 2021 Aug 23]. JAMA Intern Med. doi:10.1001/jamainternmed.2021.4260

3. Bazargan M, Bazargan-Hejazi S. Disparities in Palliative and Hospice Care and Completion of Advance Care Planning and Directives Among Non-Hispanic Blacks: A Scoping Review of Recent Literature. Am J Hosp Palliat Care. 2021;38(6):688-718. doi:10.1177/1049909120966585

4. Siler S, Arora K, Doyon K, Fischer SM. Spirituality and the Illness Experience: Perspectives of African American Older Adults. Am J Hosp Palliat Care. 2021;38(6):618-625. doi:10.1177/1049909120988280

5. Council on Ethical and Judicial Affairs. Black-white disparities in health care. JAMA. 1990;263(17):2344-2346. doi:10.1001/ jama.1990.03440170066038

\title{
Evaluation of a Digital Intervention for Hypertension Management in Primary Care Combining Self-monitoring of Blood Pressure With Guided Self-management
}

\author{
McManus RJ, Little P, Stuart B, et al. Home and Online Management and Evaluation of Blood \\ Pressure (HOME BP) using a digital intervention in poorly controlled hypertension: randomised \\ controlled trial. BMJ. 2021;372:m4858. doi:10.1136/bmj.m4858
}

\section{Study Overview}

Objective. To evaluate whether a digital intervention comprising self-monitoring of blood pressure (BP) with reminders and predetermined drug changes combined with lifestyle change support resulted in lower systolic BP in people receiving treatment for hypertension that was poorly controlled, and whether this approach was cost effective.

Design. Unmasked randomized controlled trial.

Settings and participants. Eligible participants were identified from clinical codes recorded in the electronic health records of 76 collaborating general practices from the
National Institute for Health Research Clinical Research Network, a United Kingdom government agency. The practices sent invitation letters to eligible participants to come to the clinic to establish eligibility, take consent, and collect baseline data via online questionnaires.

Eligible participants were aged 18 years or older with treated hypertension, a mean baseline BP reading of more than $140 / 90 \mathrm{~mm} \mathrm{Hg}$ and were taking no more than 3 antihypertensive drugs. Participants also needed to be willing to self-monitor and have access to the internet (with support from a family member if needed). Exclusions included BP greater than 180/110 $\mathrm{mm} \mathrm{Hg}$, atrial fibrillation, hypertension not managed by their general practitioner, chronic kidney disease stage $4-5$, 


\section{Outcomes Research in Review}

postural hypotension (>20 mm Hg systolic drop), an acute cardiovascular event in the previous 3 months, terminal disease, or another condition which in the opinion of their general practitioner made participation inappropriate.

Of the 11399 invitation letters sent out, 1389 (12\%) potential participants responded positively and were screened for eligibility. Those who declined to take part could optionally give their reasons, and responses were gained from 2426 of 10010 (24\%). The mean age of those who gave a reason for declining was 73 years. The most commonly selected reasons for declining were not having access to the internet $(982,41 \%)$, not wanting to participate in a research trial $(617,25 \%)$ or an internet study (543, 22\%), and not wanting to change drugs (535, 22\%). Of the 1389 screened, 734 were ineligible, and 33 did not complete baseline measures and randomization. The remaining 622 people who were randomized in a 1:1 ratio to receive the HOME BP intervention $(n=305)$ or usual care $(n=317)$.

Intervention vs usual care. The HOME BP intervention for the self-management of high BP consisted of an integrated patient and health care practitioner online digital intervention, BP self-monitoring (using an Omron M3 monitor), health care practitioner directed and supervised titration of antihypertensive drugs, and user-selected lifestyle modifications. Participants were advised via automated email reminders to take 2 morning BP readings for 7 days each month and to enter online each second reading. Mean home BP was calculated, accompanied by feedback of BP results to both patients and professionals with optional evidence-based lifestyle advice (for healthy eating, physical activity, losing weight if appropriate, and salt and alcohol reduction) and motivational support through practice nurses or health care assistances (using the CARE approach - congratulate, ask, reassure, encourage).

Participants allocated to usual care were not provided with self-monitoring equipment or the HOME BP intervention but had online access to the information provided in a patient leaflet for hypertension. This information comprised definitions of hypertension, causes, and brief guidance on treatment, including lifestyle changes and drugs. These participants received routine hypertension care that typically consisted of clinic BP monitoring to titrate drugs, with appointments and drug changes made at the discretion of the general practitioner. Participants were not prevented from self-monitoring, but data on self-monitoring practices were collected at the end of the trial from patients and practitioners.

Measures and analysis. The primary outcome measure was the difference in systolic BP at 12-month follow-up between the intervention and usual care groups (adjusting for baseline BP, practice, BP target levels, and sex). Secondary outcomes included systolic and diastolic BP at 6 and 12 months, weight, modified patient enablement instrument, drug adherence, health-related quality of life, and side effects from the symptoms section of an adjusted illness perceptions questionnaire. At trial, registration participants and general practitioners were asked about their use of self-monitoring in the usual care group.

The primary analysis used general linear modelling to compare systolic BP in the intervention and usual care groups at follow-up, adjusting for baseline $\mathrm{BP}$, practice (as a random effect to take into account clustering), BP target levels, and sex. Analyses were on an intention-to-treat basis and used multiple imputation for missing data. Sensitivity analyses used complete cases and a repeated measures technique. Secondary analyses used similar techniques to assess differences between groups. A within-trial economic analysis estimated cost per unit reduction in systolic BP by using similar adjustments and multiple imputation for missing values. Repeated bootstrapping was used to estimate the probability of the intervention being cost-effective at different levels of willingness to pay per unit reduction in BP.

Main results. The intervention and usual care groups did not differ significantly - participants had a mean age of 66 years and mean baseline clinical BP of 151.6/85.3 $\mathrm{mm} \mathrm{Hg}$ and 151.7/86.4 $\mathrm{mmHg}$ (usual care and intervention, respectively). Most participants were White British (94\%), just more than half were men, and the time since diagnosis averaged around 11 years. The most deprived group (based on the English Index of Multiple Deprivation) accounted for 63/622 (10\%), with the least deprived group accounting for 326/622 (52\%). 


\section{Outcomes Research in Review}

After 1 year, data were available from 552 participants (88.6\%) with imputation for the remaining 70 participants (11.4\%). Mean BP dropped from 151.7/86.4 to 138.4/80.2 $\mathrm{mmHg}$ in the intervention group and from 151.6/85.3 to $141.8 / 79.8 \mathrm{~mm} \mathrm{Hg}$ in the usual care group, giving a mean difference in systolic $\mathrm{BP}$ of $-3.4 \mathrm{mmHg}(95 \% \mathrm{Cl}-6.1$ to $-0.8 \mathrm{mmHg}$ ) and a mean difference in diastolic BP of $-0.5 \mathrm{mmHg}$ (-1.9 to $0.9 \mathrm{mmHg}$ ). Exploratory subgroup analyses suggested that participants aged 67 years or older had a smaller effect size than those younger than 67. Similarly, while the effect sizes in the standard and diabetes target groups were similar, those older than 80 years with a higher target of $145 / 85 \mathrm{mmHg}$ showed little evidence of benefit. Results for other subgroups, including sex, baseline BP, deprivation, and history of self-monitoring, were similar between groups.

Engagement with the digital intervention was high, with 281/305 (92\%) participants completing the 2 core training sessions, 268/305 (88\%) completing a week of practice BP readings, and 243/305 (80\%) completing at least 3 weeks of BP entries. Furthermore, 214/305 (70\%) were still monitoring in the last 3 months of participation. However, less than $1 / 3$ of participants chose to register on 1 of the optional lifestyle change modules. In the usual care group, a post-hoc analysis after 12 months showed that 112/234 (47\%) patients reported monitoring their own $\mathrm{BP}$ at home at least once per month during the trial.

The difference in mean cost per patient was $£ 38$ (US $\$ 51.30, € 41.9 ; 95 \% \mathrm{Cl} £ 27$ to £47), which along with the decrease in systolic BP, gave an incremental cost per $\mathrm{mmHg}$ BP reduction of $£ 11$ ( $£ 6$ to £29). Bootstrapping analysis showed the intervention had high (90\%) probability of being cost-effective at willingness to pay above £20 per unit reduction. The probabilities of being cost-effective for the intervention against usual care were $87 \%, 93 \%$, and $97 \%$ at thresholds of $£ 20$, £30, and $£ 50$, respectively.

Conclusion. The HOME BP digital intervention for the management of hypertension by using self-monitored BP led to better control of systolic BP after 1 year than usual care, with low incremental costs. Implementation in primary care will require integration into clinical workflows and consideration of people who are digitally excluded.

\section{Commentary}

Elevated BP, also known as hypertension, is the most important, modifiable risk factor for cardiovascular disease and mortality. Clinically significant effects and improvements in mortality can be achieved with relatively small reductions in BP levels. Long-established lifestyle modifications that effectively lower BP include weight loss, reduced sodium intake, increased physical activity, and limited alcohol intake. However, motivating patients to achieve lifestyle modifications is among the most difficult aspects of managing hypertension. Importantly, for individuals taking antihypertensive medication, lifestyle modification is recommended as adjunctive therapy to reduce BP. Given that target blood pressure levels are reached for less than half of adults, novel interventions are needed to improve BP control - in particular, individualized cognitive behavioral interventions are more likely to be effective than standardized, single-component interventions.

Guided self-management for hypertension as part of systematic, planned care offers the potential for improvements in adherence and in turn improved long-term patient outcomes. $^{2}$ Self-management can encompass a wide range of behaviors in addition to medication titration and monitoring of symptoms, such as individuals' ability to manage physical, psychosocial and lifestyle behaviors related to their condition. ${ }^{3}$ Digital interventions leveraging apps, software, and/or technologies in particular have the potential to support people in self-management, allow for remote monitoring, and enable personalized and adaptive strategies for chronic disease management. ${ }^{4-5}$ An example of a digital intervention in the context of guided self-management for hypertension can be a web-based program delivered by computer or phone that combines health information with decision support to help inform behavior change in patients and remote monitoring of patient status by health professionals. Well-designed digital interventions can effectively change patient health-related behaviors, improve patient knowledge and confidence for self-management of health, and lead to better health outcomes..$^{6-7}$

This study adds to the literature as a large, randomized controlled trial evaluating the effectiveness of a digital intervention in the field of hypertension and with follow-up for a year. The authors highlight that relatively few studies have been performed that combine self-monitoring with 
a digitally delivered cointervention, and none has shown a major effect in an adequately powered trial over a year. Results from this study showed that HOME BP, a digital intervention enabling self-management of hypertension, including self-monitoring, titration based on self-monitored BP, lifestyle advice, and behavioral support for patients and health care professionals, resulted in a worthwhile reduction of systolic BP. In addition, this reduction was achieved at modest cost based on the within trial cost effectiveness analysis.

There are many important strengths of this study, especially related to the design and analysis strategy, and some limitations. This study was designed as a randomized controlled trial with a 1 year follow-up period, although participants were unmasked to the group they were randomized to, which may have impacted their behaviors while in the study. As the authors state, the study was not only adequately powered to detect a difference in blood pressure, but also over-recruitment ensured such an effect was not missed. Recruiting from a large number of general practices ensured generalizability in terms of health care professionals. Importantly, while study participants mostly identified as predominantly White and tended to be of higher socioeconomic status, this is representative of the aged population in England and Wales. Nevertheless, generalizability of findings from this study is still limited to the demographic characteristics of the study population. Other strengths included inclusion of intention-to-treat analysis, multiple imputation for missing data, sensitivity analysis, as well as economic analysis and cost effectiveness analysis.

Of note, results from the study are only attributable to the digital interventions used in this study (digital webbased with limited mechanisms of behavior change and engagement built-in) and thus should not be generalized to all digital interventions for managing hypertension. Also, as the authors highlight, the relative importance of the different parts of the digital intervention were unable to be distinguished, although this type of analysis is important in multicomponent interventions to better understand the most effective mechanism impacting change in the primary outcome.

\section{Applications for Clinical Practice}

Results of this study demonstrated that among participants being treated with hypertension, those engaged with the HOME BP digital intervention (combining self-monitoring of blood pressure with guided selfmanagement) had better control of systolic BP after 1 year compared to participants receiving usual care. While these findings have important implications in the management of hypertension in health care systems, its integration into clinical workflow, sustainability, long-term clinical effectiveness, and effectiveness among diverse populations is unclear. However, clinicians can still encourage and support the use of evidence-based digital tools for patient self-monitoring of BP and guided-management of lifestyle modifications to lower BP. Additionally, clinicians can proactively propose incorporating evidence-based digital interventions like HOME BP into routine clinical practice guidelines.

\section{-Katrina F. Mateo, PhD, MPH \\ Financial disclosures: None. \\ doi:10.12788/jcom.0068}

\section{References}

1. Samadian F, Dalili N, Jamalian A. Lifestyle Modifications to Prevent and Control Hypertension. Iran J Kidney Dis. 2016;10(5):237-263.

2. McLean G, Band R, Saunderson K, et al. Digital interventions to promote self-management in adults with hypertension systematic review and meta-analysis. J Hypertens. 2016;34(4):600-612. doi:10.1097/HJH.0000000000000859

3. Bodenheimer T, Lorig K, Holman H, Grumbach K. Patient self-management of chronic disease in primary care. JAMA. $2002 \mathrm{Nov}$ 20;288(19):2469-2475. doi:10.1001/jama.288.19.2469

4. Morton K, Dennison L, May C, et al. Using digital interventions for self-management of chronic physical health conditions: A meta-ethnography review of published studies. Patient Educ Couns. 2017;100(4):616-635. doi:10.1016/j.ped.2016.10.019

5. Kario K. Management of Hypertension in the Digital Era: Small Wearable Monitoring Devices for Remote Blood Pressure Monitoring. Hypertension. 2020;76(3):640-650. doi:10.1161/ HYPERTENSIONAHA.120.14742

6. Murray E, Burns J, See TS, et al. Interactive Health Communication Applications for people with chronic disease. Cochrane Database Syst Rev. 2005;(4):CD004274. doi:10.1002/14651858.CD004274. pub4

7. Webb TL, Joseph J, Yardley L, Michie S. Using the internet to promote health behavior change: a systematic review and meta-analysis of the impact of theoretical basis, use of behavior change techniques, and mode of delivery on efficacy. J Med Internet Res. 2010;12(1):e4. doi:10.2196/jmir.1376 